\title{
NEAR-FIELD INTERACTION OF CLOSED CELLS FOR METAMATERIAL CREATION
}

\author{
Aleksandr Mironchev*, Aleksandr Gorst \\ National Research Tomsk State University, 634050, Tomsk, Russia
}

\begin{abstract}
This article presents the results of numerical and computer modeling of the flat closed conductor with different variants of arrangement. The interaction of the conductors is examined and the results of active and reactive part of the Poynting vector for each structure is presented. According to the results the model with identical parameters for each element was built and examined for the presence of metamaterial properties.
\end{abstract}

\section{Intorduction}

Back in the late 1960s, V.G. Veselago predicted the unique properties of materials with a negative real part of the permittivity and permeability [1]. Later, a special interest was aroused by the works of D. Pendry of the possibility of developing "superlenses" allowing overcoming the diffraction limit [2].

Previously, the authors of this work presented the possibility of metamaterial creation for the radio range as an artificial composite medium made up of a certain oriented linear and circular conductors $[3,4]$. Such a medium may have a negative refractive index. In the theoretical consideration of the works above stated the approach of not interacting elements has been introduced. For more complete analysis when creation of the structure with the properties of the metamaterial it is necessary to consider the influence of elements on each other at the incidence of a plane wave [5].

\section{Modelling the interaction of confined conductors}

The problem of the circular conductors' structure arrangement is sufficiently capacious. It is necessary to select geometric dimensions of the elements, their orientation, and the distance between them. To estimate the interaction between the individual elements of the array we will consider Poynting vector along the axis of distribution of the incident plane wave $[6,7]$.

To consider the active $(\operatorname{Re}(\mathrm{P}))$ and reactive $(\operatorname{Im}(\mathrm{P}))$ parts of the Poynting vector it was provided a modeling using CST Microwave studio software package. This product allows considering different characteristics of the electric $(E)$ and magnetic $(H)$ fields.

\footnotetext{
* Corresponding author: mironchev42@mail.ru
} 
For the first model a closed conductor with the outer radius $-4 \mathrm{~mm}$, inner radius -2 $\mathrm{mm}$, conductor thickness $-1 \mathrm{~mm}$ was chosen. The ring was in free space i.e. with permittivity $(\varepsilon)$ and permeability $(\mu)$ equal to unity. The plane electromagnetic wave falls to the conductor, the vector of the electric component was directed perpendicular to the axis of the ring. The estimation was performed at the frequency $F=1 \div 13 \mathrm{GHz}$.

To calculate the active and reactive parts of the Poynting vector it is required to estimate three components of the fields $E$ and $H$, such as amplitude $(|A|)$ and phase $(\varphi)$, for the further calculation of the complex field defined by the formula:

$$
B=A \cdot \exp (-i \phi)
$$

where A is amplitude of the field, $\varphi$ is phase.

Calculating the necessary characteristics and specifying the electric and magnetic fields of each projection on the coordinate axes, it becomes possible to calculate the Poynting vector by the following formula:

$$
\vec{P}=\frac{1}{2}\left[\vec{E} \times \vec{H}^{*}\right]
$$

where $\vec{E}$ is a vector of the electric field, $\vec{H}$ is a magnetic field vector.

Figure 1 shows the real part of the Poynting vector along the direction of distribution of the incident wave in the plane of the ring. The diagram is made for three frequencies $7 \div 9$ $\mathrm{GHz}$ where the maximum effect of the incident wave interaction with the circular conductor is observed. Normalization to the value of the Poynting vector modulus of the primary wave is made. The boundary of the ring is indicated by dotted lines.

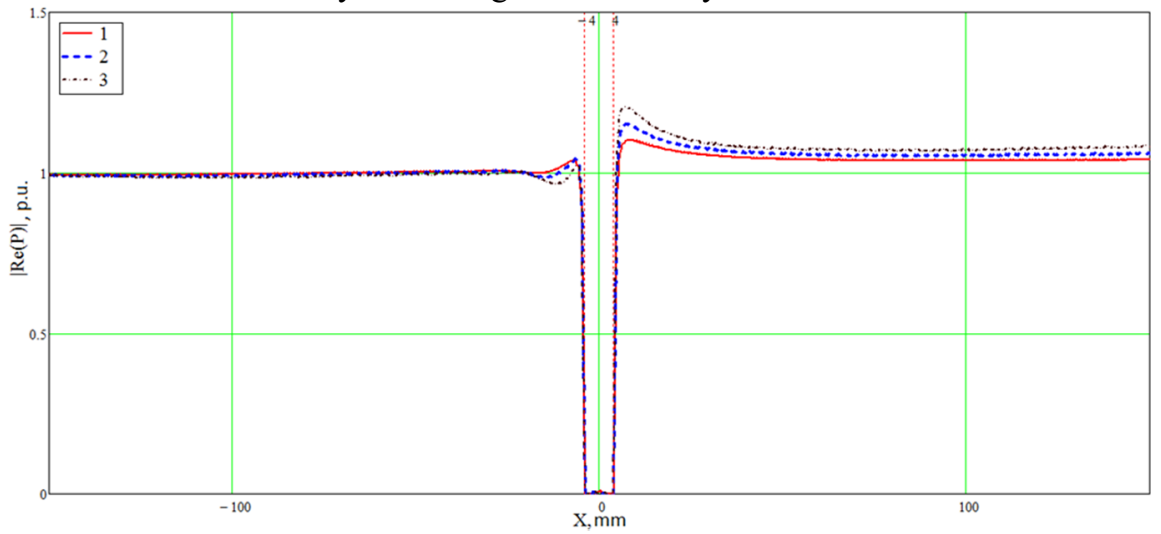

Figure 1. The real part of the Poynting vector at the plane wave incidence to the circular conductor for three frequencies: 1 - for $7 \mathrm{GHz}, 2$ - for $8 \mathrm{GHz}$ to 8,3 - for $9 \mathrm{GHz}$.

The diagram shows that at the incidence of the plane wave to the closed conductor it acts as a secondary source of electromagnetic waves. This is due to vectoring of currents on the ring. As it can be seen, the radiation is both against the direction of the incident wave distribution and co-directed with it. But it can be seen that in front of the ring the secondary field decreases rapidly as the incident wave dominates. For the conductor the effect of increasing of the Poynting vector active part is observed that is associated with the addition of the incident and the generated waves. It can also be noted that the real part of the Poynting vector (further $\mathrm{Re}(\mathrm{P})$ for simplicity) at a frequency of $9 \mathrm{GHz}$ has a maximum index. This occurs due to the approach to the resonant frequency. 
When considering the imaginary part of the Poynting vector (further $\operatorname{Im}(\mathrm{P})$ for simplicity) presented in Figure 2 it can be seen that in front of the ring there is a field of the incident wave interference with the wave generated by the ring. At the distances of about $15 \mathrm{~mm}$ from the ring in both sides the amplitude is much higher; this is due to the near zone of radiation of the wave resulting from the generation of the ring.

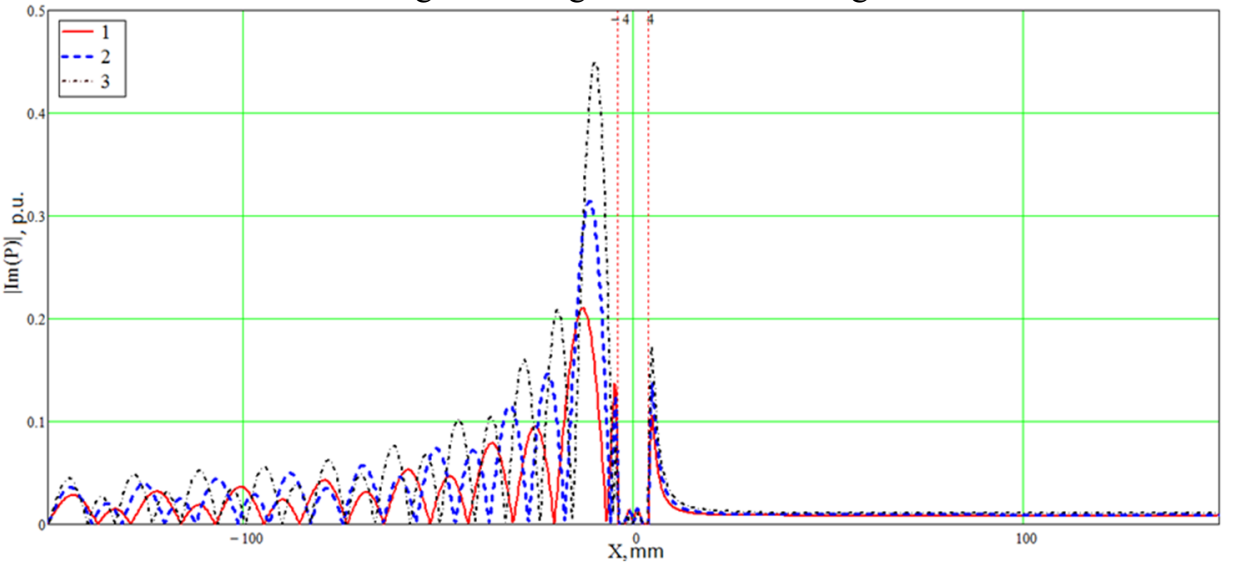

Figure 2. Imaginary part of the Poynting vector at the plane wave incidence to the circular conductor for three frequencies: 1 - for $7 \mathrm{GHz}, 2$ - for $8 \mathrm{GHz}, 3$ - for $9 \mathrm{GHz}$.

Now let's consider $\operatorname{Re}(\mathrm{P})$ and $\operatorname{Im}(\mathrm{P})$ for three closed conductors (rings) being axially (along the axis $\mathrm{OZ}$ ) as shown in Figure 3.

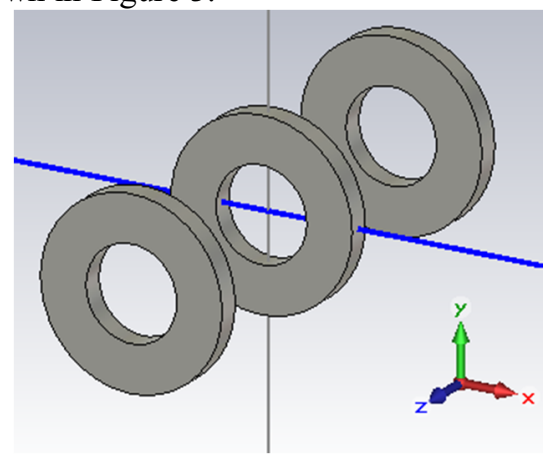

Figure 3. Model from three closed co-axial conductors.

The conductors are identical to each other and located at the distance of $5 \mathrm{~mm}$. Having the results of calculations and using formulas (1) and (2), we get $\operatorname{Re}(\mathrm{P})$ and $\operatorname{Im}(\mathrm{P})$ for three closed conductors. Figure 4 shows the comparison diagrams of the active component of the Poynting vector at the frequency of $9 \mathrm{GHz}$ of one ring with three rings. 


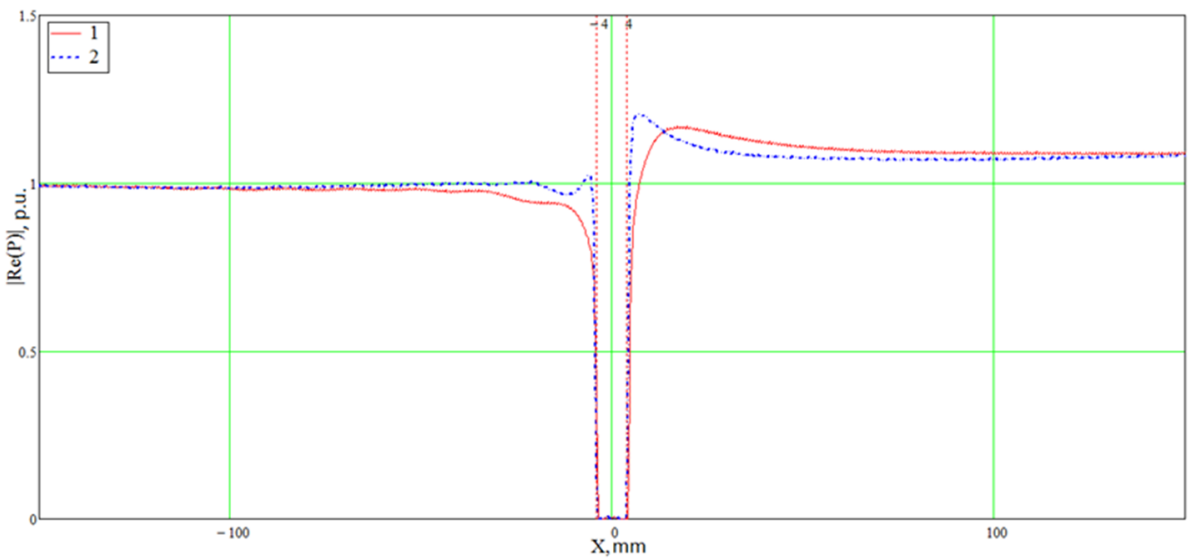

Figure 4. Real part of the Poynting vector at the frequency of $9 \mathrm{GHz}: 1$ - for three rings, $2-$ for one ring.

Figure 4 shows that the real part of the Poynting both for one ring and for the three rings is almost identical, except for some changes nearby the ring. The diagram clearly shows that the increase of the amplitude out of the conductors is happened not in a sharp jump but smoothly due to the influence of the neighboring rings.

Figure 5 demonstrates the dependence of the reactive part of the Poynting vector on the distance. In front of the conductors the behavior of the imaginary part is similar with one ring; amplitude increase is due to the presence of the rings being on both sides of the center one. But in the near field out of the rings the reactive part is significantly less. At the other frequencies at a given location of the rings the changes in the real and imaginary components of the Poynting vector are insignificant. Maximum interaction is observed at the frequency of $9 \mathrm{GHz}$.

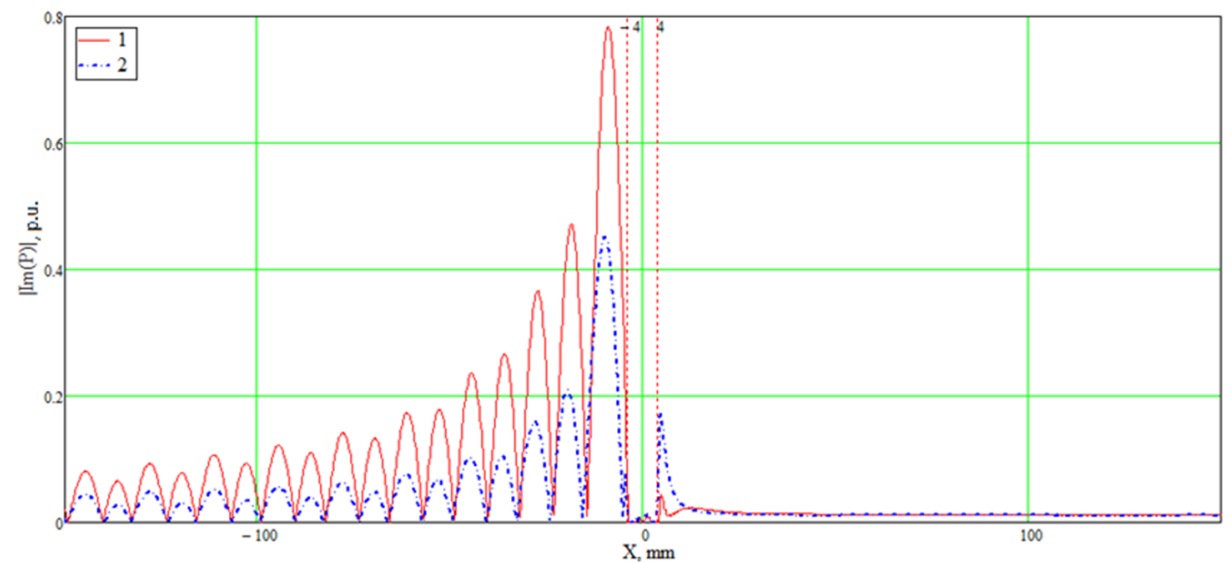

Figure 5. Reactive part of the Poynting vector at the frequency of $9 \mathrm{GHz}: 1$ - for three rings, 2 - for one ring.

Further, in Figure 6 there is a model for the case of the location of additional rings above and below (along the OY axis). E vector of the plane wave is directed along the axis OY. 


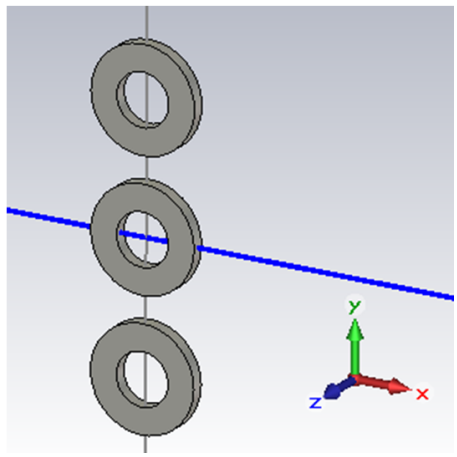

Figure 6. Model of closed conductors located along the axis OY.

The distance between the rings is $2 \mathrm{~mm}$. We also compare the result with $\operatorname{Re}(\mathrm{P})$ and $\operatorname{Im}(\mathrm{P})$ for one ring; the results are given at the frequency of $9 \mathrm{GHz}$ as in other frequencies the diagrams character is similar and maximum interaction is observed exactly at this frequency.

Diagrams for the active component of the Poynting vector are shown in Figure 7 . We can see that the amplitude of $\operatorname{Re}(\mathrm{P})$ in the case with 3 rings before the conductors up to a distance of $8 \mathrm{~cm}$ from them is equal to unity. With the approach to the rings the decrease of the amplitude associated with the reflected wave and near field generated by the conductors is observed.

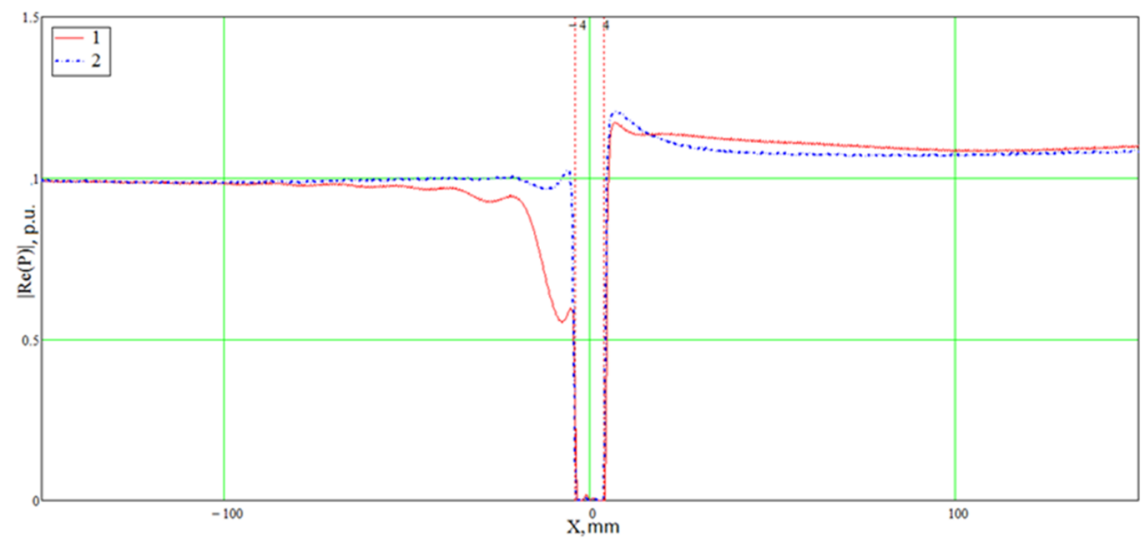

Figure 7. Active part of the Poynting vector: 1 - three rings, 2 - one ring.

Outside the rings it is evident that the active part of the Poynting vector in the near field for three rings ranks below in amplitude to one ring, but in the far field at distances from 15 $\mathrm{mm}$ to $115 \mathrm{~mm}$ there is a greater index of the real part for three rings.

Then let's compare $\operatorname{Im}(\mathrm{P})$. As shown in Figure 8 in front of the rings the imaginary part of the Poynting vector for three rings dominates, this is due to a larger area which is reflected by the incident wave, and the secondary field emitted by the conductors. Out of the elements an increase in the reactive part of the Poynting vector in the case of three rings can be noticed. Moreover, the decrease of $\operatorname{Im}(\mathrm{P})$ for three closed conductors is slower than in the case with one ring, and compared with the reaction field index for one ring at the distance of $30 \mathrm{~mm}$. 


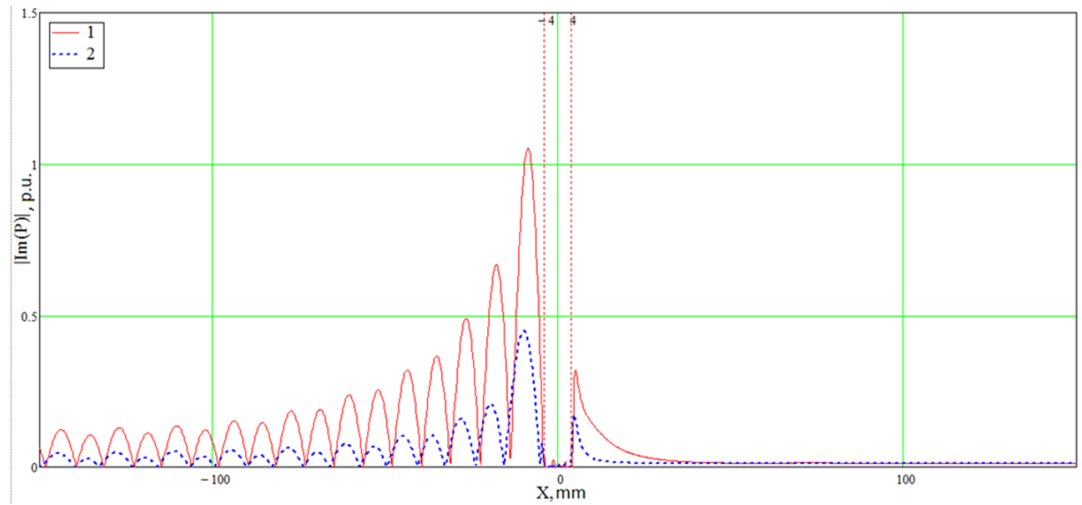

Figure 8. Reactive part of the Poynting vector: 1 -three rings, 2 - one ring.

Another possible case is shown in Figure 9, the construction of a model from three closed conductors, it is their location along the axis of distribution of the incident wave (along the axis OX).

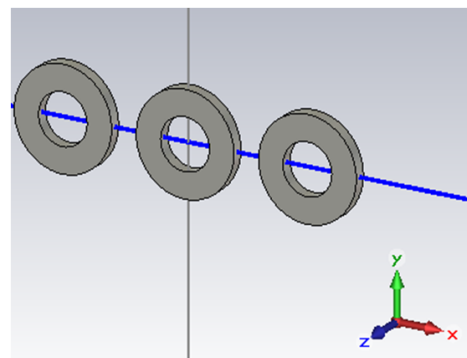

Figure 9. The model of closed conductors along the axis OX.

The distance between the rings as in the previous case is $2 \mathrm{~mm}$. As before, the interaction of three rings will be compared with one ring. The results are given at $9 \mathrm{GHz}$ as in the previous cases.

From Figure 10 it can be seen a clear predominance of the active component of the Poynting vector for three rings, which indicates a sufficiently strong interaction of the rings facing each other, but with increasing of the distance from the rings the amplitude goes up to one level. However, in front of the elements the active field is comparable.

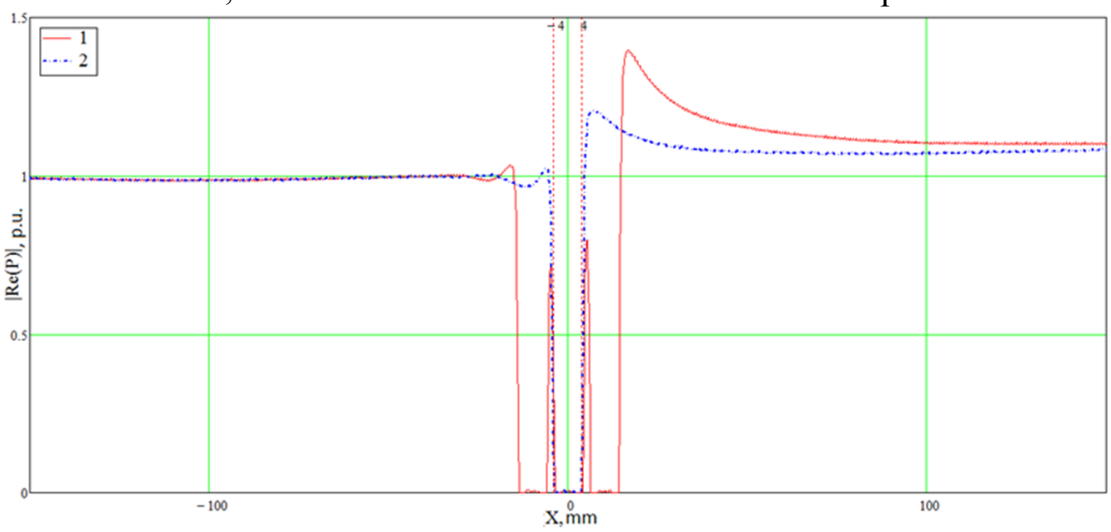

Figure 10. Active part of the Poynting vector, 1 - three rings, 2 - one ring. 


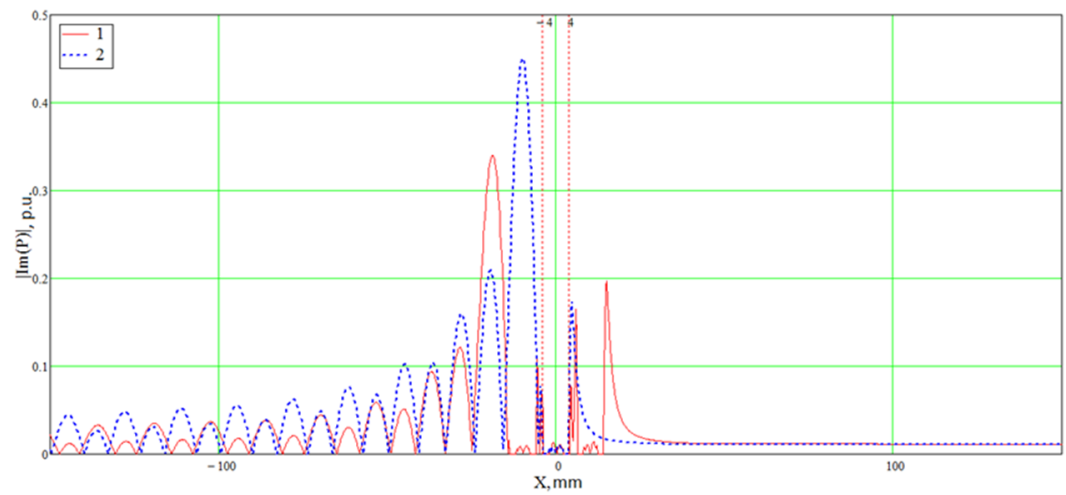

Figure 11. Reactive part of the Poynting vector: 1 - three rings, 2 - one ring.

The diagrams shown in Figure 11 illustrate the comparison of the reaction field in the case of the incidence of the plane wave to one ring element and three identical ring conductors arranged one behind another. It can be seen that for one conductor significantly higher indexes of the imaginary part of the Poynting vector to the elements than for three rings are observed. Out of the conductors the pattern is comparable both in magnitude and the rate of decay.

\section{Modeling the structure with metamaterial properties}

These results allow us to estimate the interaction of the ring elements with each other and to help in the modeling of structures in order to achieve the properties of metamaterials.

Based on all the above mentioned studies the lattice from the circular conductors with identical parameters was modeled. The lattice is consisted of four layers and had 324 elements. Because the metamaterial is characterized by a negative refractive index or a negative phase incursion in the layer, so there was this parameter.

In Figure 12 there is a diagram of the phase incursion for the lattice of the closed ring conductors at the frequency of $9 \mathrm{GHz}$. It can be seen that there is a small negative phase incursion at passing the lattice. This incursion may be counted into the effective refractive index. At this frequency its real part is $\operatorname{Re}(n)=0.344$. At the frequency of $7 \mathrm{GHz}$ it was $\operatorname{Re}(n)=-0.993$, which can be explained by a stronger interaction of elements on each other, and slightly larger near field for each of them. 


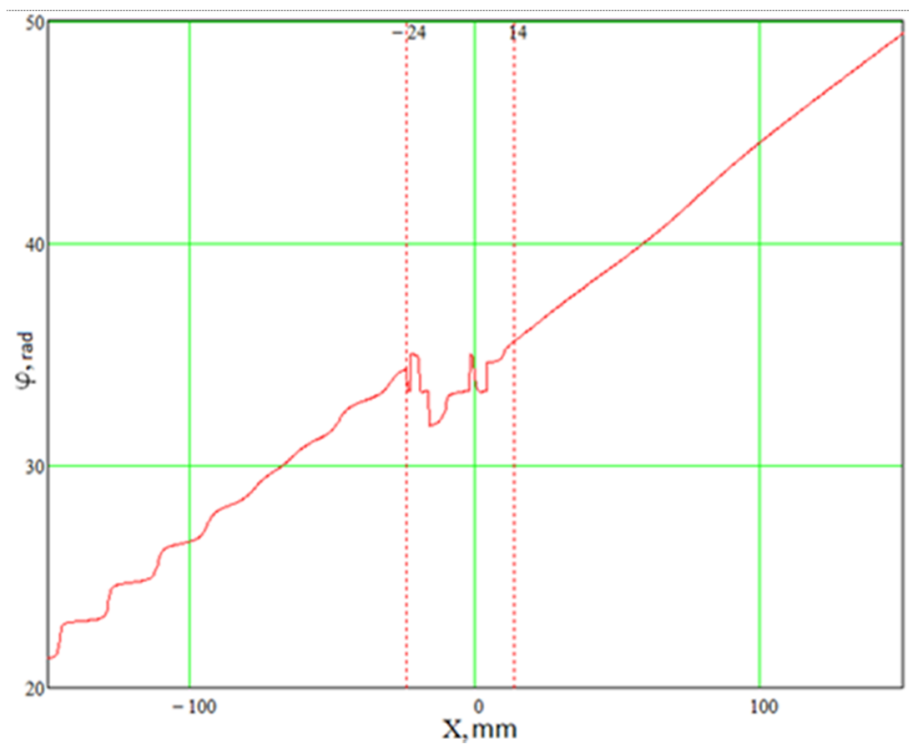

Figure 12. Phase incursion of the plane wave at the frequency of $9 \mathrm{GHz}$.

\section{Conclusion}

In view of the above stated diagrams it can be concluded that the maximum increase of the active part of the Poynting vector out of the elements can be reached at the location of the rings one after another (along the axis OX). Similar effect, but slightly smaller in size, can be reached at the location of the rings on top of each other (along the axis OY), minimal effect is observed at the location of the rings on the sides (along the axis OZ). The real part of the Poynting vector in front of the conductors is significantly reduced only in case if the rings are located above each other (along the axis OY), while in the model with rings being one after another (along the axis OX) there is a slight increase of the active field in front of the elements. In the case with the rings located on the sides (along the axis OZ) there is a decrease in the level before the conductors, but it happens quite smoothly. As for the reactive component of the field, in front of the elements it is maximum in the case of location of the rings over each other (along the axis OY), and it is minimum at the location of the rings one after another (along the axis OX). Out of the conductors the imaginary part of the Poynting vector is as maximum at the location of the rings on top of each other (along the axis OY), and minimum in the case of the rings location on the sides (along the axis OZ). At location of the rings one after another (along the axis OX) the decrease of the reactive field out of the layer is faster than at the location of the rings one above the other (along the axis OY).

Thus, by virtue of the dative analysis of the near-field interaction of the closed circular conductors with each other we were able to build a lattice model with the properties of the metamaterial.

\section{Acknowledgment}

This research carried out in 2016 was supported by «Russian Science Foundation» grant (No. 16-19-10272). 


\section{References}

[1] V.G. Veselago, Sov. Phys. Usp. 10, 509 (1968)

[2] J. B. Pendry, Physical review letters 85, 3966 (2000) doi: 10.1103/PhysRevLett.85.3966

[3] V.P. Yakubov, V.P. Belichenko, V.V. Fisanov Fundamentals of electrodynamics radiation and its interaction with the material (Publishing NTL, Tomsk, 2010)

[4] V.P. Yakubov, A.S. Mironchev, A.G. Andreytsov, I.O. Ponomareva, Russian physics journal 53 , 895 (2011) doi: 10.1007/s11182-011-9507-5

[5] J.B. Pendry, D.R. Smith, Physics Today 57, 37 (2004)

[6] I.V. Lindell, A.H. Sihvola , Electromagnetic Waves and Applications 19, 861 (2005) doi: 10.1163/156939305775468741

[7] Dan Xiaa, Dianyou Song and Zhiyong Wang, MATEC Web of Conferences 61, 4 (2016) doi: 10.1051/matecconf/20166102004 